\title{
NUEVAS ESTRATEGIAS \\ EDUCATIVAS EN EL ÁREA \\ DE LAS COMUNICACIONES
}

Mar de Fontcuberta*

* Directora del Magíster en Comunicación Social de la Facultad de Comunicaciones de la Pontificia Universidad Católica de Chile. 


\section{RESUMEN}

El primer desafío para la educación del siglo XXI es un cambio de actitud. Frente a una mirada tradicional que concibe la enseñanza como transmisión de conocimientos, se impone un nuevo concepto, el de gestión del conocimiento, entendido como la capacidad de obtener las informaciones necesarias, saber seleccionarlas, articularlas y aplicarlas a un determinado campo.

La educación terciaria no está ajena a este proceso. El aumento de programas de diplomado, maestría y doctorado en el mundo y en Chile obedece a la aparición de nuevas exigencias en la sociedad, con objetivos y diseños más ajustados a los nuevos tiempos. En respuesta a tales requerimientos, la Pontificia Universidad Católica de Chile creó dos nuevos tipos de maestría en el área de las comunicaciones.

\section{ABSTRACT}

The first challenge for education in the $21^{\text {st }}$. century is a change of attitude. Faced with a traditional approach that views education as a communication of knowledge, a new concept emerges, that of the management of knowledge, i.e. the ability to obtain the necessary information, to be able to select it, and to organize it and apply it to a given field.

Tertiary education is also part of this process The increasing number of diploma-track courses, master's degree and doctor's degree programs in Chile and on a world-wide level is a response to new demands by society, with goals and designs which are better adapted to the times at hand. In reply to such requirements, the Pontificia Universidad Católica de Chile has created two new types of master's degrees in the area of communications. 


\section{NUEVAS ESTRATEGIAS EDUCATIVAS EN EL ÁREA DE LAS COMUNICACIONES}

\section{CONSIDERACIONES GENERALES}

Los estudios de posgrado han sufrido grandes transformaciones en los últimos años. El aumento de programas de diplomado, maestría y doctorado en el mundo y en Chile es síntoma de la aparición de nuevos requerimientos de formación en la sociedad. Estas necesidades exigen una oferta más plural, con objetivos y diseños más ajustados a los nuevos tiempos. Entre los factores que configuran esta situación se encuentran: los continuos cambios que se producen en el ámbito de los conocimientos y el crecimiento enorme del volumen de información disponible; la necesidad de especialización, profundización y una constante actualización de conocimientos en un campo específico; el aumento de la demanda de formación en nuevas disciplinas en el ámbito académico y profesional; $y$, finalmente, la necesidad creciente de establecer objetos de estudio desde una perspectiva multidimensional y, por lo tanto, transdisciplinaria.

A lo anterior podemos sumar dos factores más: la progresiva globalización que afecta de modo significativo a la universidad y que se traduce en un intercambio académico que rebasa las fronteras del campo de la docencia y la investigación; y, por otra parte, la introducción de las nuevas tecnologías que posibilitan la enseñanza y el aprendizaje a través de las redes.

Hay un punto de partida clave: lo que hoy llamamos conocimiento es algo distinto de lo que así se ha considerado tradicionalmente y que, en gran parte, se sigue impartiendo en universidades. "Son tres los factores que han precipitado el cambio: a) el crecimiento enorme 
del volumen de la información disponible y los continuos cambios que experimenta el conocimiento; $b$ ) las demandas del sistema económico y social, y $c$ ) la progresiva socialización de las nuevas tecnologías en el acceso, transmisión y recepción de la información" (Fontcuberta, 1997: 272).

Actualmente es imposible almacenar un acervo de conocimientos que duren para toda la vida. La cantidad de información es tan impresionante como el poco tiempo que tarda en quedar obsoleta por el descubrimiento de nuevos datos que complementan o sustituyen a los obtenidos en investigaciones anteriores. La puesta al día de saberes ha de ser constante, y para ello hay dos respuestas posibles que el profesor Antonio Bartolomé define así: "o la necesidad de una permanente actualización, o la necesidad de diseñar y utilizar nuevos modos de organizar y acceder a la información" (Bartolomé). Creo que lo primero no niega lo segundo. Eso significa que la persona va a tener que dedicar una parte fundamental de su vida profesional y académica a la actualización, es decir, a la formación continua, que implica la necesidad de cursar programas de perfeccionamiento (de posgrado en el caso que nos ocupa), y a hacerlo desde parámetros distintos a los de la tradición universitaria vigente.

En efecto, hasta hace muy poco se ha identificado el conocimiento con la acumulación de saberes, memorizada, ordenada, clasificada, jerarquizada y sancionada según un canon preestablecido que sigue determinadas pautas espacio-temporales. De acuerdo con ellas, el alumno debe recorrer con esfuerzo un camino en el que va a adquirir sucesivamente, es decir, de modo secuencial, una serie de conocimientos impartidos por varios profesores en un espacio determinado (clase), que seguirán sus respectivos programas según un diseño curricular y que, una vez superadas las distintas pruebas establecidas al efecto, le conducirán a la obtención de un título reconocido socialmente que lo identificará como poseedor de los conocimientos necesarios para ser experto en un ámbito determinado del saber. Sin embargo, como afirma Bartolomé: "Conocer es hoy algo más que ser capaz de reproducir nombres, hechos y conceptos. Pues de día en día crece la distancia entre lo que somos capaces de recordar y el volumen total de la información (...). Todo esto nos lleva a una idea clave: la enseñanza debe cambiar. En estos momentos nos hemos 
fijado en dos aspectos: la menor importancia que debe darse a la reproducción de conocimientos, y la mayor importancia que debe darse al desarrollo de destrezas en el acceso a la información" (Bartolomé). El primer desafío que debe afrontar la educación del siglo XXI es un cambio de actitud. Frente a una identificación tradicional de la educación como transmisión de conocimientos, se impone un nuevo concepto: el de gestión del conocimiento. Pero, ¿qué significa gestionar el conocimiento? "En principio acceder a las informaciones necesarias, saber seleccionarlas, articularlas y aplicarlas a un determinado objetivo. (...) Eso significa que el nuevo conocimiento exige una formación distinta en el campo profesional y laboral ya que cada vez un empleado se valora más por su capacidad de acceso a la información, de evaluación, análisis y solución de problemas, que por la simple acumulación de saber, por extensa que sea. Es más importante la capacidad de un profesional de autoorganizarse con eficacia que de seguir las instrucciones al pie de la letra. Todo ello ha de ser tenido en cuenta por el sistema educativo" (Fontcuberta, 2001: 48).

Lo anteriormente expuesto supone una revolución que plantea una idea clave: hay que dar prioridad al desarrollo de las aptitudes cognitivas de orden superior de quienes aprenden. "Estas aptitudes pueden agruparse en tres grandes categorías: el conocimiento profundizado de cierta esfera del saber, la comprensión de las condiciones en las cuales se puede utilizar ese saber, y, por fin, una facultad de control y de pilotaje metacognitivo. Es decir, la enseñanza debe tener un contenido; el saber debe poder utilizarse para resolver problemas incluso en situaciones nuevas, no exploradas, y, por último, un individuo debe tener el control consciente y voluntario sobre sus propios procesos cognitivos, sobre sus razonamientos, sobre la manera en que ese individuo aprende, sobre lo que ocurre en su memoria y sobre el modo en que puede mejorar su funcionamiento para mejorar los resultados" (Délacôte p. 108).

El ámbito laboral también está cambiando en varios aspectos que el sistema educativo no siempre tiene en cuenta. De acuerdo con el profesor Jack Treuhaft, estos factores son los siguientes:

- Las empresas e instituciones buscan empleados que sean competentes en información y tecnología. 
- El nuevo conocimiento exige una educación para los empleados que ponga el énfasis en la capacidad de acceso a la información, solución de problemas, análisis, evaluación y toma de decisiones.

- Los cambios rápidos y la incertidumbre del puesto de trabajo hacen que las empresas e instituciones busquen personas que ya tengan las habilidades adquiridas.

- Los estudiantes necesitan formación a corto plazo, pues así lo exigen los continuos cambios en el conocimiento.

- El volumen creciente de trabajadores a tiempo parcial requiere un acceso más flexible a la educación.

- La expansión del conocimiento en todos los campos es tan rápida que supera toda capacidad individual para ponerse al día. Saber cómo acceder a la información, más que memorizarla, es básico para enfrentarse a ese continuo reciclaje.

- Hay una tendencia decreciente en el número de estudiantes jóvenes y progresiva respecto de la cantidad de alumnos mayores. (Treuhaft).

La respuesta académica a todos esos desafíos ha sido desigual. Un problema bastante extendido es que la universidad no siempre ha reconocido el hecho de que actualmente los estudios de posgrado, en especial en el campo de las ciencias sociales, no deben orientarse en forma prioritaria al desarrollo de la carrera académica y que es necesario flexibilizar la tipología de la oferta. La progresiva complejidad de la sociedad obliga a coexistir a dos procesos aparentemente (solo aparentemente) contradictorios: la profundización en un campo concreto (especialización) y la referencia de ese campo a un contexto más amplio que lo abarca y lo explica (transdisciplinariedad). El primero supone la aparición de estudios de maestría de carácter profesional muy especializado; el segundo, la creación de posgrados interdisciplinarios en nuevos ámbitos de estudio (un ejemplo lo constituye la bioética). Las características de unos y otros son distintas. Hay universidades que se han querido poner a la vanguardia ofreciendo programas nuevos con una actitud que responde más al concepto de mercadeo que al de la excelencia académica. En algunos casos, las innovaciones han sido ejecutadas sin la necesaria reflexión y, sobre todo, sin evaluar los resultados. En otros, no se ha considerado que los nuevos conocimientos exigen también cambios en la metodología docente. Ello se ha producido, por ejemplo, cuando se han introducido 
las nuevas tecnologías en la educación sin tener en cuenta que los nuevos soportes han reconfigurado la figura del alumno, del profesor y de todo el proceso de acceso al conocimiento. En otros casos más, finalmente, se han implementado las necesarias modificaciones en los programas tradicionales o se han creado nuevos programas con la reflexión, el desarrollo y el debido rigor académico.

La Facultad de Comunicaciones de la Pontificia Universidad Católica de Chile (PUC) ofrece un Magíster en Comunicación Social con dos menciones: una en Dirección y Edición Periodísticas, y la otra en Comunicación y Educación. La primera es el prototipo de una maestría profesional especializada; la segunda contempla un campo nuevo desde una perspectiva transdisciplinaria. Ambos programas operan en un mismo contexto: el de la relación de la sociedad con los medios de comunicación, y aunque lo hacen desde ángulos diferentes, son complementarios.

\section{MAGÍSTER EN DIRECCIÓN Y EDICIÓN PERIODÍSTICAS}

El Magíster en Dirección y Edición Periodísticas es un posgrado dirigido tanto a personas especializadas en el área como a profesionales y licenciados provenientes de otras disciplinas que tengan o hayan tenido vinculación con el quehacer informativo y que podrían a futuro realizar tareas directivas en los medios de comunicación. Su propio desarrollo ha ido configurando un perfil de alumno(a) que requiere una elevada especialización para las dos funciones periodísticas señaladas. Dicha especialización es una clara demanda social y de mercado que, por razones obvias, no se obtiene en el pregrado.

La creación del programa respondió a un análisis de las oportunidades externas determinadas por varios factores, entre los que cabe destacar los cambios constantes en el mundo empresarial y profesional del sector que obligan a un reciclaje permanente, la necesidad de enfatizar comportamientos profesionales concordantes con principios éticos, y la diversificación del campo laboral que ha llevado a muchos periodistas a crear sus propias empresas, sobre todo en el campo de la producción multimedial y audiovisual. Las actuales licenciaturas de periodismo no contemplan la figura del periodistaempresario, y por lo tanto existe un claro vacío en ese campo. 
El programa tiene como objetivo general promover una instancia de reflexión académica que permita desarrollar, en un profesional, la capacidad de evaluar críticamente los medios de comunicación, de emitir juicios sobre el proceso informativo y de tomar decisiones tanto empresariales como editoriales. Se centra en dos grandes áreas: a) producción y edición del proceso informativo, y b) gestión de la empresa informativa. Sus contenidos buscan desarrollar la capacidad de editar y difundir un producto periodístico de calidad de acuerdo a principios éticos, y la adopción de una perspectiva empresarial en el sector de los medios de comunicación en todas sus variantes, desde la dirección comercial hasta los recursos humanos.

La maestría tiene una duración de cinco trimestres. Uno de los problemas que ha tenido que enfrentar es el de conseguir armonizar el desarrollo sistemático de una actividad académica con alta exigencia de dedicación y los vaivenes propios de la profesión periodística, a la que pertenece la inmensa mayoría de los alumnos y que tiene como característica un horario laboral muy variable. No es lo mismo trabajar en un diario matutino que vespertino, ni en un programa televisivo o radial que se emite por la mañana o por la noche. Ello ha obligado a cambiar los horarios varias veces. En ese sentido la respuesta de las empresas periodísticas ha sido de diverso tipo. En algunos casos han ofrecido becas o han dado facilidades para que sus periodistas pudieran estudiar; en otros, sin embargo, los interesados han tenido problemas para seguir las actividades lectivas. En todo caso, la mayoría de los alumnos cursa los estudios asumiendo una responsabilidad económica.

Otro de los problemas que el posgrado ha debido afrontar es la insuficiente percepción por parte de algunos postulantes de lo que significa un máster especializado. Debido al interés que motivan los medios de comunicación y los contenidos informativos que difunden, en más de un caso licenciados de otras carreras han pretendido encontrar en la maestría la entrada a un campo que siempre les había resultado atractivo, o ciertos conocimientos periodísticos (entendidos simplemente como herramientas) que solucionarían ausencias en su formación de origen. A veces ha sido difícil hacer entender que un máster con mención en Dirección y Edición Periodísticas no enseña reporteo y que, si bien profundiza en algunos contenidos de la 
Licenciatura de Periodismo, su meta es una especialización, que persigue objetivos muy concretos y basados en las demandas sociales y de mercado de un contexto social específico.

Es precisamente el análisis continuo de esa demanda lo que llevó a introducir modificaciones en los contenidos y la estructura tras unos años de experiencia. En el primer caso, se incorporaron materias que reforzaran el área de gestión (Dirección Comercial, Dirección de Recursos Humanos, Creación de Proyectos o Casos de Gestión de Medios), y otras destinadas a introducir nuevos aspectos del área editorial (Edición en Convergencia). Con respecto a la estructura, a partir del año 2003 se implantó el sistema trimestral de cursos. Una de las razones para ello fue la necesidad de fomentar y facilitar la interdisciplina con otros posgrados trimestrales de la PUC (en especial con los que tienen que ver con el MBA o con Administración). Además, al acortar la duración de los cursos, se posibilita la incorporación de nuevos ramos que profundicen en las diversas materias.

Otro de los objetivos de este último cambio fue crear las condiciones favorables para recibir a profesores extranjeros cuya presencia ha sido constante y cuyo aporte se ve como fundamental. La experiencia ha permitido constatar que gran parte de esos docentes podía venir a nuestro país en los meses de julio y agosto, lo que obligaba a comprimir demasiado sus exposiciones. Un inconveniente adicional es que en esa época se desarrollan las vacaciones de invierno, por lo que resultaba complicado programar algunas invitaciones. El sistema trimestral permite combinar las visitas de los profesores a lo largo de los distintos trimestres aprovechando, por ejemplo, las vacaciones de invierno o de primavera que tienen lugar en las universidades del hemisferio norte.

Esta maestría es la única de su modalidad en Chile y, hasta donde hemos podido saber, en el resto de América Latina. Una de sus fortalezas es que ha contado desde su primera edición con la presencia de alumnos extranjeros; otra, no desdeñable, es que la mayoría de ellos, al regresar a sus países, ha encontrado puestos de dirección o ha mejorado sustancialmente sus condiciones laborales. La presencia de alumnos de otras nacionalidades también ha sido una de las constantes del otro posgrado que exponemos, el Magíster en Comunicación y Educación. 


\section{MAGÍSTER EN COMUNICACIÓN Y EDUCACIÓN}

El Magíster en Comunicación Social mención en Comunicación y Educación es un posgrado de carácter profesional que busca responder, desde una perspectiva transdisciplinaria, a las necesidades formativas que plantean las nuevas vinculaciones entre comunicación y educación. Este máster se propone formar profesionales que sean capaces tanto de poner los medios de comunicación al servicio de la comunicación educativa, como de adaptar contenidos educativos para incrementar y potenciar, de mejor manera, el aprovechamiento educativo de los medios de comunicación. El programa depende del Instituto de Estudios Mediales de la Facultad de Comunicaciones de la PUC, que está concebido como un espacio para reforzar la relación de los medios de comunicación con otras disciplinas. En su diseño participó un equipo de académicos de las facultades de Comunicaciones y de Educación de la Universidad Católica.

El máster es actualmente el único existente en Chile que abarca y articula el proceso completo de análisis, reflexión, uso, diseño, producción, gestión y evaluación de contenidos educativos para los medios de comunicación. Está dirigido en especial a licenciados en comunicación social que deseen diseñar, producir o gestionar productos educativos en los medios; a licenciados en educación interesados en formarse en el conocimiento profundo de los medios que requiere la Reforma Educativa chilena; a periodistas que se desempeñen o estén interesados en el ámbito de la educación, y a licenciados provenientes de otras disciplinas que necesiten desarrollar tareas en el campo de la comunicación y educación (campañas educativas y políticas de comunicación pública, entre otras opciones). Este posgrado pretende responder a la actual demanda de contenidos y productos de calidad y en español, tanto para los sistemas de comunicación tradicionales, como para los nuevos medios, a saber, multimedia interactivos, redes telemáticas e internet ${ }^{1}$. Estos últimos, en particular, se distinguen por las enormes posibilidades educativas que brindan a través de su gran versatilidad y flexibilidad, su carácter globalizador y la capacidad de integrar lenguajes con enormes potencialidades expresivas.

1 Es necesario considerar que, en la actualidad, los contenidos generales en español disponibles en la red no superan el cinco por ciento del total, y que los propiamente educativos no superan el 0,5 por ciento. 
El programa aborda cinco grandes áreas:

1. Perspectivas teóricas en el campo de la comunicación educativa y su aplicación en diversos procesos e instrumentos.

2. Desarrollo, manejo y creación de contenidos educativos para los medios de comunicación.

3. Uso de los medios de comunicación en la educación formal y no formal.

4. Producción y gestión de medios de comunicación educativos.

5. Investigación en el campo de la comunicación y educación.

Existen dos características que hacen de esta maestría un caso especial: la primera es que cuenta con un convenio suscrito con la Universidad Autónoma de Barcelona (UAB), mediante el cual los alumnos de la PUC tienen la posibilidad de cursar en forma paralela y complementaria, a lo largo de tres semestres, el mismo posgrado dictado por la universidad española y obtener así ambos grados sin necesidad de desplazarse y sin costo adicional de arancel. La segunda es que introduce una concepción metodológica innovadora, que combina las clases presenciales con actividades a través de las redes para reforzar el proceso de aprendizaje del alumno y el trabajo en equipo. Además, cuenta con un sistema de tutoría individualizada durante todo el transcurso de los estudios.

El acuerdo con la UAB implica una organización bastante compleja, tanto en el ámbito administrativo como en el docente. Cada estudiante es alumno de pleno derecho de ambas universidades, lo que supone duplicidad de matrículas pero no de contenidos. El máster de la PUC es presencial y el de la UAB se imparte en modalidad semipresencial. La universidad española envía cada semestre a varios profesores que imparten clases presenciales en régimen concentrado a lo largo de una semana, con cursos complementarios al programa de la universidad chilena. La maestría de la UAB también ofrece parte de sus contenidos por internet a través de un sitio web al que puede accederse con clave personalizada y donde se encuentran bases de datos, desarrollo de los temas, bibliografía, foros, modalidades de ejercicios y análisis de casos prácticos. De esta forma el alumno puede elegir entre una enorme variedad de contenidos y perspectivas, pues tiene posibilidades de interactuar con un número importante de profesores extranjeros de primer nivel: ventajas muy difíciles de obtener 
en otras circunstancias. Asimismo, si lo prefiere y cuenta con los recursos necesarios, puede desplazarse a la universidad española para realizar su proyecto de tesis de grado.

Uno de los aspectos más relevantes en la metodología docente en este programa es la redacción por parte del estudiante de una memoria. Es un documento tipo "bitácora" elaborado a lo largo de todo el posgrado, que recoge de modo sintético los grandes temas de las asignaturas, sus aportaciones a las mismas y cómo esas materias han sido incorporadas a sus prácticas profesionales y a su proyecto de vida. La filosofía de la memoria radica en que el alumno reflexione y registre diariamente sus ideas y experiencias desde su realidad y sus intereses.

Se trata de un trabajo progresivo, constante y personal que debe cumplir con todos los requisitos y rigor académico que exige el posgrado. Su calificación forma parte del proyecto final y su última entrega pasa también por la Comisión Evaluadora del Proyecto de Tesis de Grado. El objetivo de la memoria es reflejar el proceso de aprendizaje personal y vivencial realizado por el alumno bajo la mirada especial de los conocimientos adquiridos en ambas maestrías (de la PUC y de la UAB) y bajo el prisma de su proyecto en particular. A ese respecto hay que señalar que tanto la memoria como el proyecto de tesis serán los mismos para las dos universidades. Los dos trabajos, además, serán evaluados por ambas instituciones.

Al inicio del posgrado se designa un tutor para cada alumno, quien tendrá la labor de realizar el seguimiento de su proceso de aprendizaje y guiarlo en el desarrollo de su memoria en sus distintas entregas. La memoria es el testimonio escrito de un proceso diario que implica cambios, por lo cual no tiene sentido relegar su elaboración a un resumen final.

El Magíster en Comunicación y Educación de la PUC entiende la memoria como un instrumento que media entre el proceso de enseñanza y el aprendizaje del alumno. Esta estrategia surge a partir de un concepto esencial en la educación actual: la metacognición, es decir, "la capacidad de pensar el pensamiento, la capacidad de controlar y orientar voluntariamente por uno mismo sus propios procesos de tratar la información; todo esto dentro del marco de una toma de 
conciencia de la existencia de tales procesos. Una enseñanza que tenga esta dimensión es aquella que permite al alumno interiorizar la crítica del proceso de resolución de problemas, pasando por la crítica exterior que hace el enseñante a una autocrítica. Esta modelización que hace el alumno del control cognitivo del maestro y luego su interiorización pueden compararse con el andamiaje que facilita la construcción de un edificio; los andamios se retiran una vez terminada la construcción. El andamiaje sirve para construir el aprendizaje del exterior y luego, progresivamente, es desmantelado a medida que el alumno va adquiriendo una autonomía crítica" (Délacôte p. 137).

\section{ALGUNAS CONSIDERACIONES EN TORNO A LA EXPERIENCIA}

El Magíster en Comunicación y Educación se encuentra en su tercera versión. Una de sus características es la enorme variedad disciplinaria de sus alumnos, que abarca desde periodistas y educadores a licenciados en teatro, guionistas de televisión, sicólogos y diseñadores gráficos. Una mezcla que aumenta la complejidad del objeto estudiado pero que se ve enriquecida por la heterogeneidad de puntos de vista. Es todavía pronto para tener una perspectiva suficiente y valorar los resultados de la implantación de la memoria. Sin embargo, ya se trabaja en una propuesta de evaluación para poder aplicarla a las próximas versiones del programa. De todas formas, y aunque no pueda considerarse todavía un diagnóstico, sí se puede apuntar algunos aspectos reveladores constatados en su desarrollo, los cuales tienen que ver sobre todo con la falta de experiencia de los estudiantes al enfrentarse con una metodología nueva y sin ningún referente. Así, la mayoría de los alumnos tiene dificultades para entender el concepto del aprendizaje como "proceso". En general, cuando llegan, consideran la maestría como un depósito de contenidos que les conviene aprender, como un paréntesis en sus vidas y un medio para conseguir objetivos de tipo profesional y laboral. En segundo lugar, acostumbrados a las rutinas externas imperantes en el sistema educativo (pruebas, lecturas, recensiones, exámenes), los estudiantes no tienen el hábito de reflexionar -a partir de un instrumento como la memoria- sobre sus procesos cognitivos.

Muchos de ellos experimentan una clara separación entre lo que viven y lo que estudian y tienden a mantener ambas experiencias en ámbitos separados. No tienen claro qué tipo de vivencias personales y 
profesionales pueden incorporar a la memoria y, cuando lo hacen, sobre todo al principio, es con gran inseguridad. Asimismo, a menudo tienen dificultad para articular las distintas experiencias vividas en diferentes instancias (laborales, profesionales, académicas, vitales) en un solo documento y con un determinado propósito. También les cuesta aplicar, desde el primer día, lo que aprenden a lo que viven y analizar los resultados.

Sin embargo, al cabo de algunos meses puede verificarse un cambio notable en la mayoría de las memorias. Por una parte, se asume que es un instrumento válido como estrategia de aprendizaje, y en ese sentido dichos trabajos cada vez más constan de dos partes claramente diferenciadas: una común a todos los alumnos, integrada por los contenidos del currículo y que solo difiere a la hora de plantear el enfoque de su tratamiento de los distintos temas; y otra personal, autónoma, que progresivamente incorpora las experiencias externas al máster vividas por el estudiante en su contexto y que, a partir de una reflexión teórica, se traduce a menudo en la incorporación a la memoria de guiones de televisión y radio, actividades educomunicativas en las aulas de colegios, institutos o centros de formación profesional, propuestas de actuación en capacitación laboral, actividades en comunas o municipios, y otros. Quizás sirva como referencia el texto con el que un alumno concluyó una de las primeras entregas:

"Este magíster es el inicio de un nuevo momento en mi vida, y no quiero desperdiciar la oportunidad de formarme de la mejor manera como un profesional apto para llevar a cabo el gran reto que plantea este posgrado, de comunicar un nuevo tipo de mensaje de una nueva manera.

Quiero salir de esta experiencia capacitado para actuar en el campo de la comunicación y educación, reforzando lo que sé hacer pero a la vez ampliando mis posibilidades de actuación, pues en un mundo globalizado hacen falta profesionales con un horizonte más amplio y con capacidades de actuación en diversas áreas afines.

Mis expectativas, que eran un sueño al inicio del semestre, empiezan a concretarse en un proyecto de vida que marcará un cambio importante en mi vida. Aprovecho para agradecer a mis maestros y tutores por ayudarme en esta aventura." 


\section{REFERENCIAS BibliográFicAS y NotAS}

Bartolomé, A. "Preparando un nuevo modo de conocer". EDUTEC: Revista electrónica de tecnología educativa $\mathrm{N}^{\circ}$ 4. Islas Baleares, Universidad de las Islas Baleares con la colaboración de la Asociación de Usuarios Españoles de Satélites para la Educación (EOS), Departamento Ciencias de la Educación, 1996.

Délacôte, G. Enseñar y aprender con nuevos métodos. La revolución cultural de la era electrónica. Barcelona: Gedisa, 2000.

Fontcuberta, M. "El rol de los medios de comunicación ante un nuevo tipo de conocimiento". Retos de la sociedad de la información. Salamanca: Universidad Pontificia de Salamanca-Bibliotheca Salamanticensis, 1997.

Fontcuberta, M. "Comunicación y educación: una relación necesaria". Cuadernos de Información $\mathrm{N}^{\circ}$ 14. Santiago, Pontificia Universidad Católica de Chile, Facultad de Comunicaciones, 2001.

Treuhaft, J. Changes in Education. Algonquin College of Applied Arts and Technology. Versión electrónica, 1995. 
